Article

\title{
Interface-Controlled Pd Nanodot-Au Nanoparticle Colloids for Efficient Visible-Light-Induced Photocatalytic Suzuki-Miyaura Coupling Reaction
}

\author{
Eunmi Kang, Hyeon Ho Shin and Dong-Kwon Lim *
}

KU-KIST Graduate School of Converging Science and Technology, Korea University, 145 Anam-ro, Seongbuk-gu, Seoul 02841, Korea; cool3519@naver.com (E.K.); sinhh10@gmail.com (H.H.S.)

* Correspondence: dklim@korea.ac.kr; Tel.: +82-2-3290-4611

Received: 22 September 2018; Accepted: 13 October 2018; Published: 17 October 2018

check for updates

\begin{abstract}
Plasmonic nanostructures can be employed for performing photocatalytic reactions with visible-light illumination involving two different possible mechanisms, namely, the near-field enhancement and/or direct hot-electron transfer to the conduction band of an active catalyst. In this study, we demonstrate the significant contribution of a graphene interface layer present between plasmonic nanoparticles and active catalysts (Pd nanodots) in enhancing the photocatalytic efficiency of $\mathrm{Pd}$ nanodots through an accelerated electron transfer process. The well-defined Pd-nanodot-modified gold nanoparticles with or without a graphene interface layer were prepared using a wet-chemical synthetic method. The role of the graphene interface was investigated by performing wavelength-dependent reduction studies using potassium hexacyanoferrate (III) in the presence of Pd-nanodot-modified cysteamine-modified AuNPs (Pd-cys-AuNPs), Pd-nanodot-modified graphene oxide (GO)-coated AuNPs (Pd-GO-AuNPs), and Pd-nanodot-modified reduced GO (rGO)-coated AuNPs (Pd-rGO-AuNPs). The fastest rate for the reduction of $\mathrm{Fe}^{3+}$ to $\mathrm{Fe}^{2+}$ was obtained with Pd-rGO-AuNPs because of the fast electron transfer achieved in the presence of the reduced graphene oxide layer. The highest catalytic activity for the visible-light induced C-C coupling reaction was obtained with Pd-rGO-AuNPs, indicating the role of the graphene interface layer. These results indicate that the design and use of engineered interfaces are of importance to achieve enhanced catalytic activity with plasmonic hybrid nanomaterials.
\end{abstract}

Keywords: graphene interface; visible light; photocatalyst; Suzuki-Miyaura coupling reaction

\section{Introduction}

The Suzuki-Miyaura cross-coupling reaction, which is a powerful one-step pathway of catalytic C-C bond formation, involves the coupling of an organoboron reagent and an organic halide employing thermal activation energy in the presence of a palladium (Pd) catalyst and a base [1-4]. Homogeneous catalysts with a high efficiency have been used in typical Suzuki-Miyaura cross-coupling reactions, but the difficulty in separating homogeneous catalysts has been regarded as a main limitation [1,5]. On the contrary, heterogeneous catalysts (i.e., Pd nanoparticles) can be easily separated from the reaction mixture after the reaction [6-8], but the limitation of the heterogeneous catalyst is attributed to its low activity at room temperature, thereby requiring the use of elevated temperatures or higher amounts of catalysts as compared with a homogeneous catalyst [9]. The development of visible-light-responsive heterogeneous catalysts is one of the feasible ways to overcome the low catalytic efficiency of heterogeneous catalysts [10-14]. In this regard, the incorporation of plasmonic nanoparticles with active catalysts is an interesting strategy to utilize visible-light and subsequently improve the catalytic activity of the active catalyst [15-18]. Two different mechanisms are known to 
be involved in this process. The local electromagnetic field generated on the plasmonic nanoparticle surface accelerates the production of $\mathrm{e}^{-}-\mathrm{h}^{+}$pairs in the active photocatalyst, or the hot electrons of plasmonic nanoparticle generated by light absorption can be directly transferred to the conduction band of active photocatalysts [10,19-21]. In the hot electron transfer mechanism, the intrinsic ultrafast relaxation of hot electrons in the plasmonic nanoparticle can compete with the electron transfer process occurring from the plasmonic nanoparticle to active sites because of the extremely transient lifetime of hot electrons ( $<50 \mathrm{fs}$ ) $[19,22,23]$. The possible physical and intrinsic energy barrier at the junction between plasmonic nanoparticles and active catalysts is also a critical barrier in the hot electron transfer process $[10,15,24,25]$. Due to the complex nature of plasmonic-induced photocatalysts and the limitation of the methods for investigating the role of the interface layer between the plasmonic nanoparticle and the active catalyst, the role of an interface layer in determining the photocatalytic activity has not yet been thoroughly investigated [26].

In this study, we used a graphene layer as the interface engineering material between plasmonic nanoparticles and active catalysts, owing to its variable band gap and exceptional electron mobility of $2 \times 10^{5} \mathrm{~cm}^{2} \cdot \mathrm{V}^{-1} \mathrm{~s}^{-1}$, which depend on the oxidation state of grapheme $[27,28]$. We hypothesized that the use of graphene as an interface material would facilitate the transfer of hot electrons from plasmonic nanoparticles to the active catalyst by minimizing the energy barrier between heterogeneous elements (Au vs. Pd). We used a wet-chemical synthetic method to prepare Pd-nanodot-modified AuNPs with or without the graphene layer and compared the photocatalytic activity and performance for the Suzuki C-C coupling reaction at various reaction parameters (Figure 1).
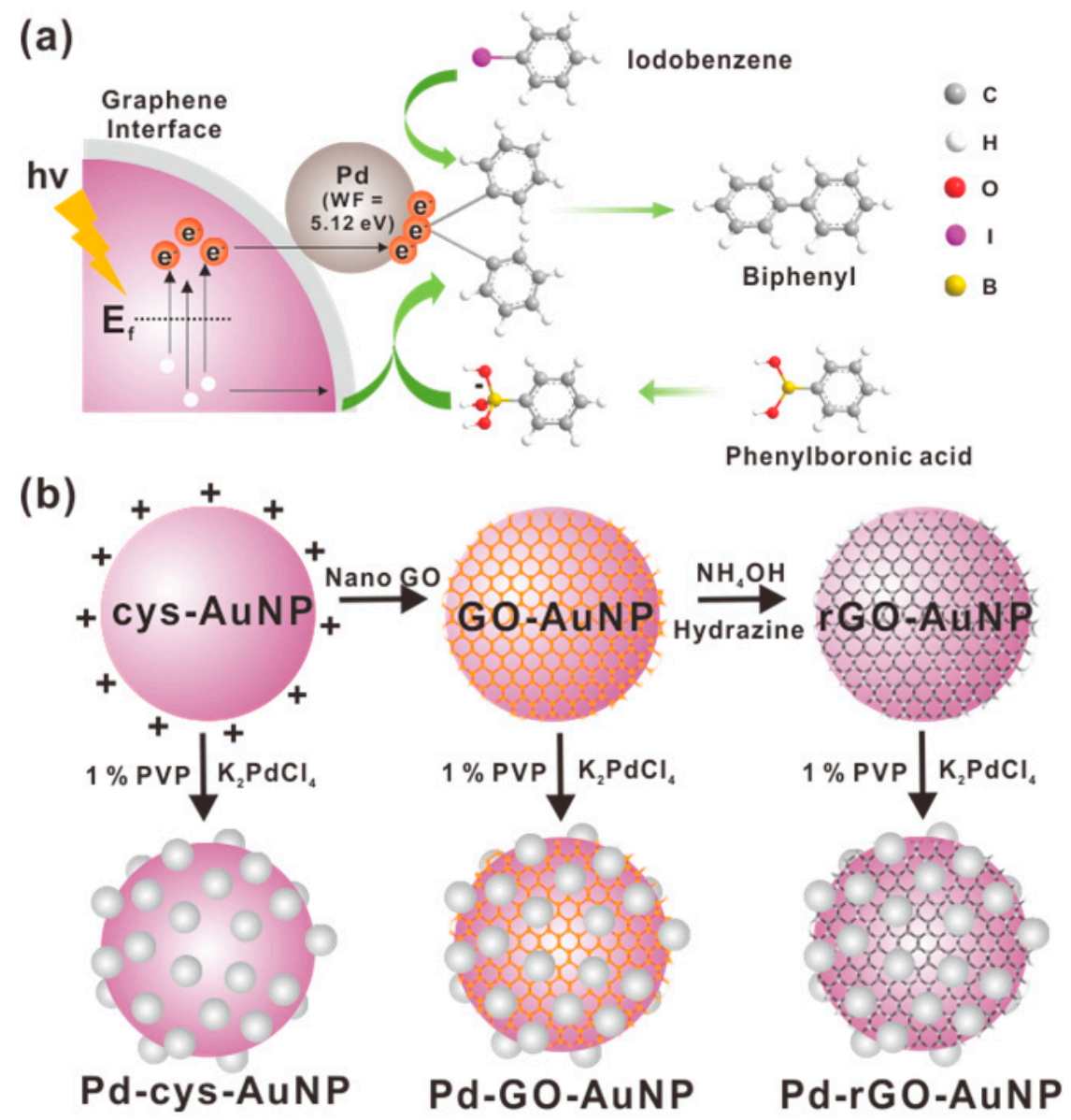

Figure 1. (a) Schematic representation of the Suzuki-Miyaura cross-coupling reaction of Pd-nanodot-decorated AuNPs with graphene interface, (b) synthetic scheme of Pd-cys-AuNPs, Pd-GO-AuNPs, and Pd-rGO-AuNPs. 


\section{Results and Discussion}

\subsection{Synthesis and Characterization of Pd-Modified-Au Nanoparticles with Graphene Interface}

No graphene interface is present in the Pd-cys-AuNPs since the Pd precursor $\left(\mathrm{K}_{2} \mathrm{PdCl}_{4} \cdot \mathrm{H}_{2} \mathrm{O}\right)$ was reduced in the presence of cys-AuNPs, which has a positively charged surface. The positively charged surface is not desirable for preparing Pd-nanodot-decorated cys-AuNPs. Interestingly, we found that the addition of polyvinylpyrrolidone (PVP) into the reaction mixture resulted in the formation of Pd-nanodots on cys-AuNPs (Figures S2 and S3). On using citrate-AuNPs as a seed for Pd-nanodot decoration instead of cys-AuNPs, Pd-nanodots were successfully formed on the surface of citrate-AuNPs regardless of the presence of PVP as shown in Figure S3. This could be attributed to the preference of $\mathrm{Pd}^{2+}$ precursors for the negatively charged surface [29]. For comparison, we employed cys-AuNPs as a core to prepare Pd-nanodot-decorated GO-AuNPs and rGO-AuNPs. The addition of PVP also helped in the formation of Pd-nanodot-decorated GO-AuNPs and rGO-AuNPs. The prepared solutions of Pd-cys-AuNPs, Pd-GO-AuNPs, and Pd-rGO-AuNPs exhibit red wine, purple, and blue colors, respectively, with a slight broadening of the UV-vis spectra (Figure 2a and inset). Raman spectra analysis indicated the presence of a GO layer on GO-AuNPs and rGO-AuNPs with different chemical states [30]. The ratio of intensities of the D peak $\left(1346.9 \mathrm{~cm}^{-1}\right)$ to the $G$ peak $\left(1600 \mathrm{~cm}^{-1}\right)$ in the Raman spectrum of Pd-GO-AuNPs (green line in Figure $2 b$ ) was 0.53 , and this ratio was changed to 0.84 in the case of Pd-rGO-AuNPs (blue line in Figure 2b). Furthermore, transmittance electron microscope (TEM) analysis indicated the formation of Pd-nanodots $(\sim 2-3 \mathrm{~nm}$ in diameter) on the AuNPs, GO-AuNPs, and rGO-AuNPs (Figure 2c-e and ). The element mapping analysis based on TEM images clearly indicated the distribution of Pd (green) on AuNPs (blue) (Figure 2c-e). The presence of carbon (red) from GO or rGO could be clearly identified on or around the AuNPs (Figure 2d,e). X-ray diffraction (XRD) analysis revealed the presence of characteristic peaks of $\mathrm{Au}(111,200,220$, 311) and Pd (222) (Figure S5). The amount of Pd on the AuNPs in the Pd-cys-AuNPs, Pd-GO-AuNPs, and Pd-rGO-AuNPs was quantified to be $7.69,8.66$, and $7.60 \mathrm{mg} / \mathrm{kg}$, respectively, by employing the induced-coupled plasma analysis. These results indicate the formation of same amount of Pd-nanodots on AuNPs, GO-AuNPs, and rGO-AuNPs. 
(a)

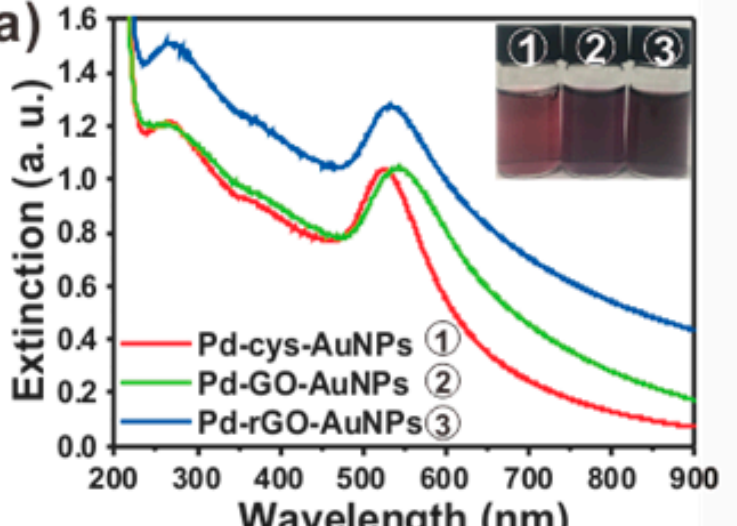

(c)

(d)
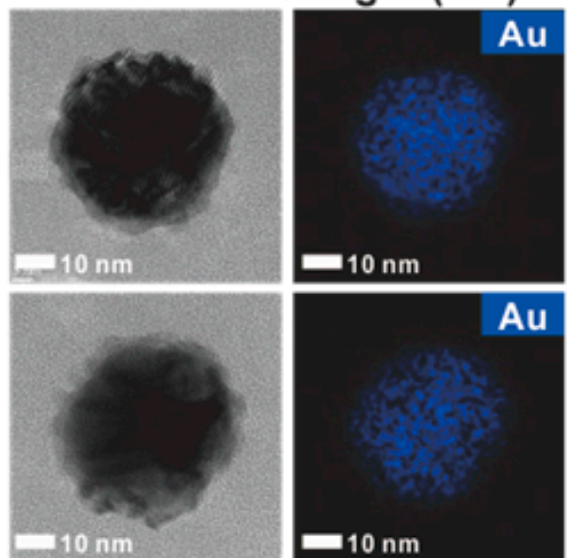

(e)

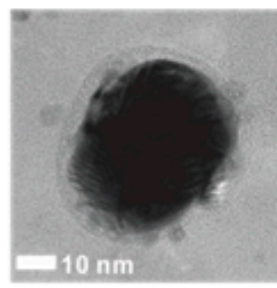

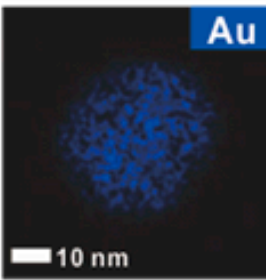

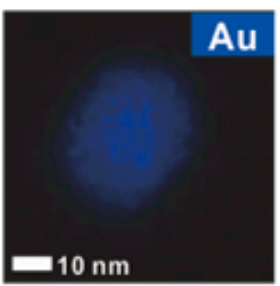

(b)
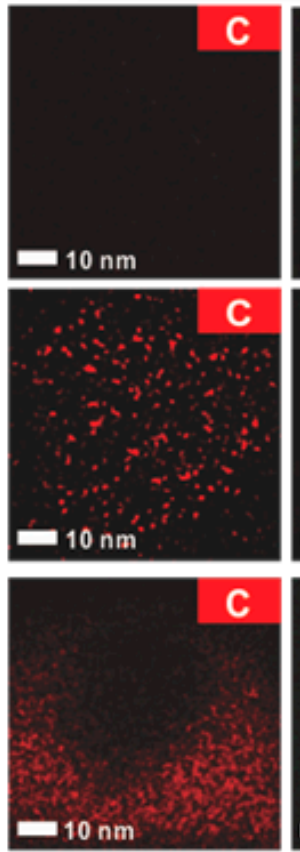
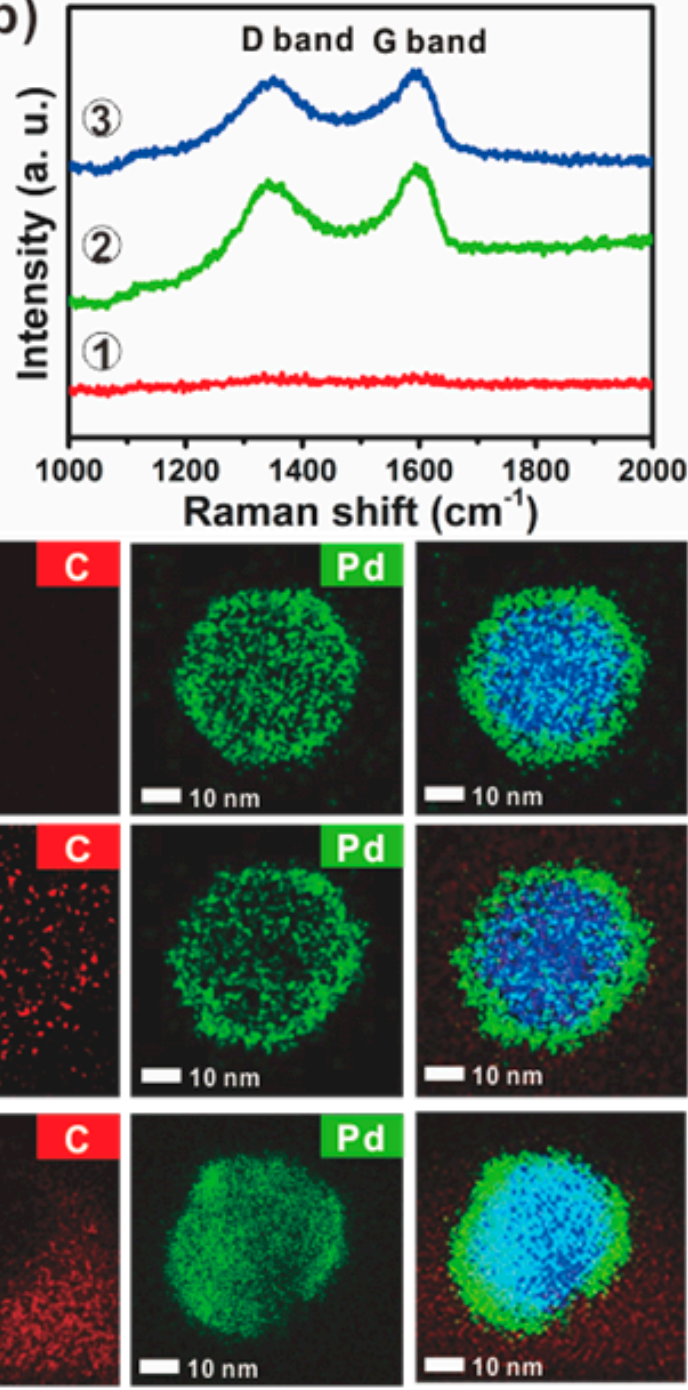

Figure 2. UV-visible spectra (inset: photograph of the solutions of as prepared catalysts), Raman spectra, and TEM images of (1) Pd-cys-AuNPs, (2) Pd-GO-AuNPs, and (3) Pd-rGO-AuNPs. (a) UV-Visible Spectra and (b) Raman spectra of Pd-cys-AuNPs (red line), Pd-GO-AuNPs (green line), and Pd-rGO-AuNPs (blue line). (c-e) TEM images and energy dispersive X-ray mapping of (c) Pd-cys-AuNPs, (d) Pd-GO-AuNPs, and (e) Pd-rGO-AuNPs.

\subsection{Time-Dependent $\mathrm{Fe}^{3+}$ Reduction Study with Visible Light and Near-Infrared Light Source}

Initially, the efficiency of the photocatalytic activity of Pd-cys-AuNPs, Pd-GO-AuNPs, and Pd-rGO-AuNPs could be quantitatively compared by examining the reduction chemistry of $\mathrm{Fe}^{3+}$ into $\mathrm{Fe}^{2+}$ [31]. As shown in Figure 3a and Figure S6, the reduction of $\mathrm{Fe}^{3+}$ to $\mathrm{Fe}^{2+}$ can be monitored by observing the changes in the peak intensity at $420 \mathrm{~nm}$, which is the characteristic peak of $\mathrm{Fe}^{3+}$. Two different light sources, namely, xenon (Xe)-lamp $\left(400-700 \mathrm{~nm}, 5.24 \mathrm{~W} / \mathrm{cm}^{2}\right)$ and near-infrared (NIR) laser $\left(808 \mathrm{~nm}, 11.4 \mathrm{~W} / \mathrm{cm}^{2}\right)$, were used to investigate the effect of the catalyst on the $\mathrm{Fe}^{3+}$ reduction efficiency while maintaining a solution temperature of $25^{\circ} \mathrm{C}$ (Figure S1). With the Xe-lamp illumination, the slowest changes in the $420 \mathrm{~nm}$ peak intensity were observed using Pd-cys-AuNPs as the photocatalyst. Pd-rGO-AuNPs exhibited the fastest reduction rate, indicating the highest photocatalytic activity among the examined Pd-Au nanoparticles (Figure $3 \mathrm{~b}$ and Figure S6). With the NIR laser $(808 \mathrm{~nm})$, no significant changes in the $\mathrm{Fe}^{3+}$ peak intensity was observed because of the presence of the non-resonant plasmonic band of AuNPs at $808 \mathrm{~nm}$ (Figure $3 \mathrm{c}$ and Figure S7). In a wavelength-dependent reduction study performed with three different excitation light sources (450, 
500 , and $630 \mathrm{~nm}$ ), the fastest reduction rate could be obtained when illuminated at $500 \mathrm{~nm}$ because of the strong plasmonic absorption of AuNPs observed at $500 \mathrm{~nm}$ (Figure 3d and Figure S8), indicating the significant role of plasmonic contribution to the reduction of $\mathrm{Fe}^{3+}$ into $\mathrm{Fe}^{2+}$.

(a)
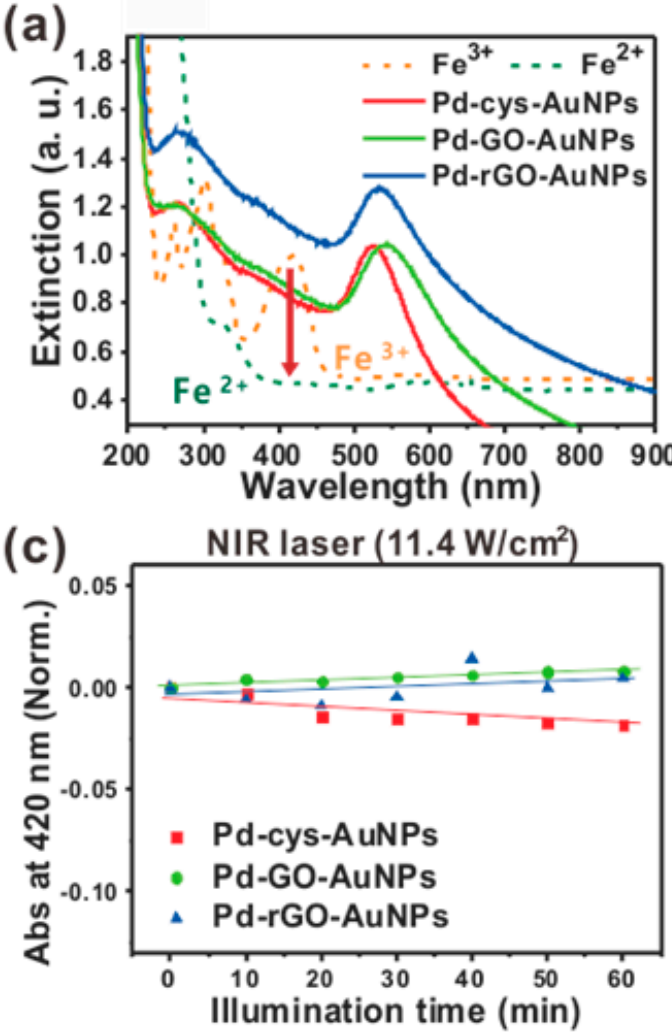

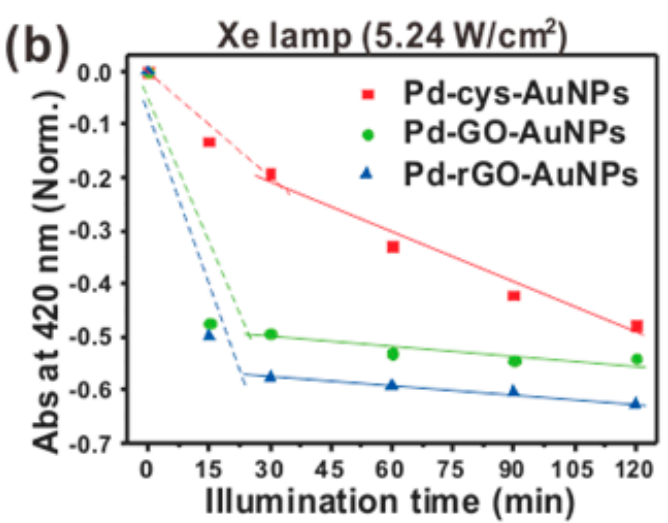

(d)

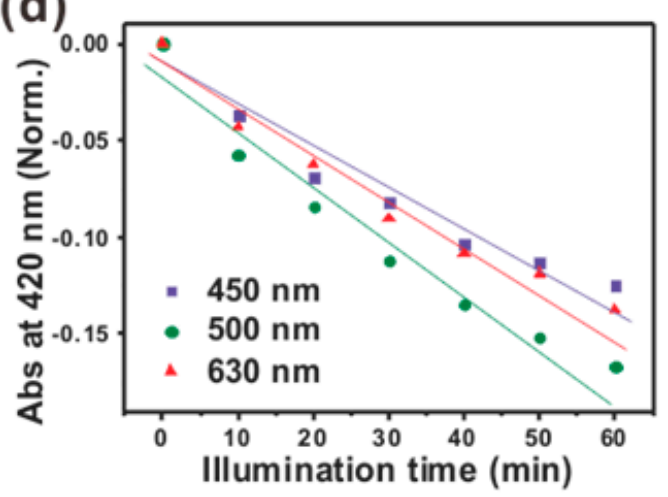

Figure 3. Kinetic study of $\mathrm{Fe}^{3+}$ reduction with Pd-modified AuNPs performed with two different light sources (Xe-lamp and NIR $(808 \mathrm{~nm})$ ) and three different wavelengths. (a) UV-Visible spectra of potassium hexacyanoferrate (III) (orange line, $500 \mu \mathrm{M}$ ) in the presence of Pd-cys-AuNPs, Pd-GO-AuNPs, and Pd-rGO-AuNPs (Abs 1.0 or 1.2 at $540 \mathrm{~nm}$ ). (b,c) Time-dependent transformation of $\mathrm{Fe}^{3+}$ to $\mathrm{Fe}^{2+}$ monitored at $420 \mathrm{~nm}$ by illuminating with (b) Xe-lamp or with (c) NIR laser (808 nm). (d) Wavelength-dependent transformation of $\mathrm{Fe}^{3+}$ to $\mathrm{Fe}^{2+}$ in the presence of Pd-rGO-AuNPs (purple square, $450 \mathrm{~nm}$; green circle, $500 \mathrm{~nm}$; red triangle, $630 \mathrm{~nm}$ ).

\subsection{Photocatalytic Suzuki-Miyaura Cross-Coupling Reactions at Various Conditions}

Two contributions from the light absorption of plasmonic nanoparticles are mainly responsible for the enhanced photocatalytic coupling reactions [15]. As the Suzuki-Miyaura cross-coupling reaction could be accelerated by thermal activation energy, the strong photothermal effect of plasmonic nanoparticles might cause an enhancement in the photocatalytic reactions [32,33]. The electron-hole pair transfer to the active catalyst can also be a possible mechanism for the plasmonic photocatalytic reaction [34,35]. To clearly differentiate the significance of these two different contributions, we performed photocatalytic reactions with or without controlling the reaction temperature. The Pd-Au nanoparticle solution ( $5 \mathrm{~mL}$, optical density (OD) at $540 \mathrm{~nm}=0.33$ or 0.4 ) containing iodobenzene $(0.4 \mathrm{mmol})$, phenylboronic acid $(0.4 \mathrm{mmol}), \mathrm{K}_{2} \mathrm{CO}_{3}(800 \mathrm{mM})$, and ethanol $(6.25 \mathrm{~mL})$ was placed in a reactor (Pyrex, window diameter $=11 \mathrm{~mm}$ ) equipped with a water circulation jacket (Figure S1). The solution was irradiated with a Xe-lamp (power density: $5.24 \mathrm{~W} / \mathrm{cm}^{2}$ ). Two different conditions, namely, (1) Xe-lamp illumination without controlling the reaction temperature, (2) Xe-lamp illumination by maintaining the reaction temperature of $25^{\circ} \mathrm{C}$, were employed, and the results are summarized in Figure 4 and Figure S9. The representative chromatogram for the reaction is displayed in Figure S10. 
Figure 4a shows the results obtained from the condition (1). Pd-rGO-AuNPs (blue line) exhibited the fastest reaction progress with the yield reaching up to $66.4 \%$. On the contrary, Pd-cys-AuNPs (red line) exhibited the slowest reaction progress by achieving the lowest yield of the coupling product $(30 \%)$. The reaction attained with Pd-GO-AuNPs was faster than that with Pd-cys-AuNPs and slower than that with Pd-rGO-AuNPs. Pd-rGO-AuNPs exhibited a two times faster reaction as compared with Pd-cys-AuNPs, indicating the significant contribution of the graphene interface to the photocatalytic reaction. The final temperature of the reaction mixture after carrying out light illumination for $2 \mathrm{~h}$ was measured to be Pd-cys-AuNPs $\left(50.3^{\circ} \mathrm{C}\right)$, Pd-rGO-AuNPs $\left(51.0^{\circ} \mathrm{C}\right)$, and Pd-rGO-AuNPs $\left(52.0^{\circ} \mathrm{C}\right)$, respectively (Figure S11). In spite of the slightly higher final temperature of the reaction mixture containing Pd-rGO-AuNPs, the higher yield ( $>2$ times) of the C-C coupling product achieved with Pd-rGO-AuNPs cannot be explained based on a simple photothermal effect-based mechanism. In order to exclude the thermal effect completely to the reaction progress, the temperature of the reaction solution was maintained at $25{ }^{\circ} \mathrm{C}$ during light illumination (condition (2)). In this condition (Figure $4 \mathrm{~b}$ ), although the overall yield is lower than that achieved with the reaction carried out without controlling the reaction temperature (condition (1)), Pd-rGO-AuNPs exhibited the highest yield among other Pd-Au nanostructures (Figure $4 \mathrm{~b}$ and Figure S9; Pd-cys-AuNPs: 6.7\%, Pd-GO-AuNPs: 30.9\%, and Pd-rGO-AuNPs: $54.5 \%$ ). This result indicates the significant contribution of electron-transfer mechanism from plasmonic nanoparticle to active Pd nanodot catalyst via graphene interface, which is further validated with a series of experiments in the next section.
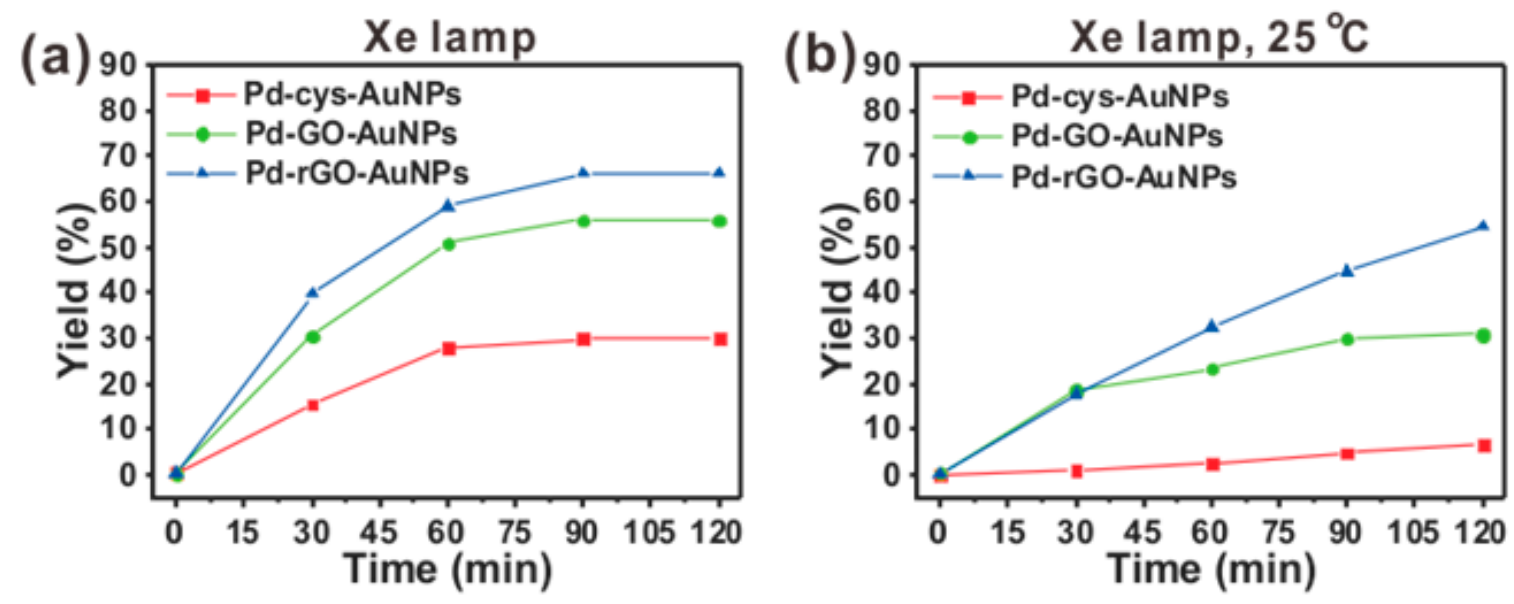

Figure 4. Comparison of the catalytic performances of Pd-cys-AuNPs, Pd-GO-AuNPs, and Pd-rGO-AuNPs for the Suzuki C-C coupling reaction at various conditions. (a) Xe-lamp illumination without controlling the reaction temperature, (b) Xe-lamp illumination by maintaining the reaction temperature of $25^{\circ} \mathrm{C}$.

\subsection{Suzuki-Miyaura Cross-Coupling Reaction under Three Different Wavelengths}

To investigate the plasmonic contributions for the enhanced photocatalytic activity, we performed wavelength-dependent Suzuki-Miyaura cross-coupling reactions in the presence of Pd-rGO-AuNPs (OD 0.4 at $530 \mathrm{~nm}$, Xe-lamp, $25^{\circ} \mathrm{C}$ ) and with three different light sources (460, 575, and $630 \mathrm{~nm}$, $\left.0.06 \mathrm{~W} / \mathrm{cm}^{2}\right)$ using a band pass filter $(20 \mathrm{~nm}$ band width) as shown in Figure 5a. The fastest reaction rate was achieved with the $575 \mathrm{~nm}$ light illumination (green line in Figure 5b, final yield: $59 \%$ ) as compared with that achieved with $460 \mathrm{~nm}$ (blue line in Figure 5b, final yield: 41\%) and $630 \mathrm{~nm}$ light illuminations (red line in Figure 5b, final yield: $39 \%$ ). This could be attributed to the presence of the plasmonic absorption peak of Pd-rGO-AuNPs at $530 \mathrm{~nm}$. The results indicate that the plasmonic light absorption is the most critical factor determining the enhancement in the photocatalytic activity of Pd-rGO-AuNPs. 


\subsection{Mechanism Study}

We performed the Suzuki-Miyaura cross-coupling reaction (Xe-lamp, $25^{\circ} \mathrm{C}$ ) with Pd-rGO-AuNPs to investigate the effect of a well-known electron scavenger, $\mathrm{KBrO}_{3}$, and a hole scavenger, triethanolamine (TEA), on the reaction progress (Figure 5c). The Pd-rGO-AuNP solution was mixed with phenylboronic acid $(48.8 \mathrm{mg}, 0.4 \mathrm{mmol})$, ethanol $(6.25 \mathrm{~mL})$, iodobenzene $(44.5 \mu \mathrm{L}, 0.4 \mathrm{mmol})$, $\mathrm{K}_{2} \mathrm{CO}_{3}(1.25 \mathrm{~mL}, 1 \mathrm{mmol})$, and electron and hole scavengers, $\mathrm{KBrO}_{3}(0.4 \mathrm{mmol})$ and TEA $(1 \mathrm{~mL})$, respectively. We found that the addition of $0.4 \mathrm{mmol} \mathrm{KBrO}_{3}$ caused a significant decrease in the yield from $67 \%$ to $39 \%$. The addition of TEA $(1.0 \mathrm{~mL})$ caused the complete quenching of the reaction as shown in Figure $5 c$ (red line). Both the results indicate that the generation of electron-hole pairs more strongly affects the photocatalytic Suzuki-Miyaura cross-coupling reaction as compared to the photothermal contributions.

(a)

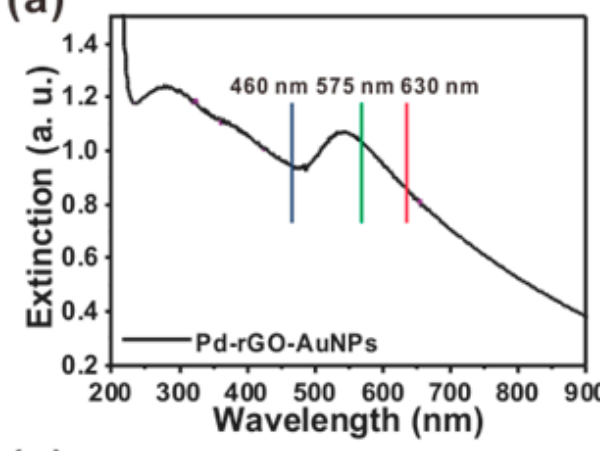

(c)

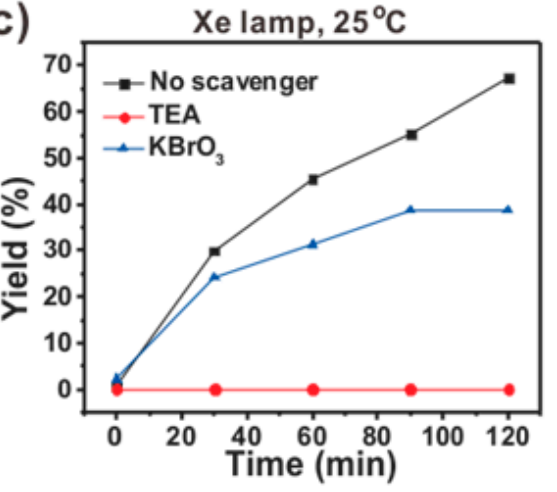

(b)

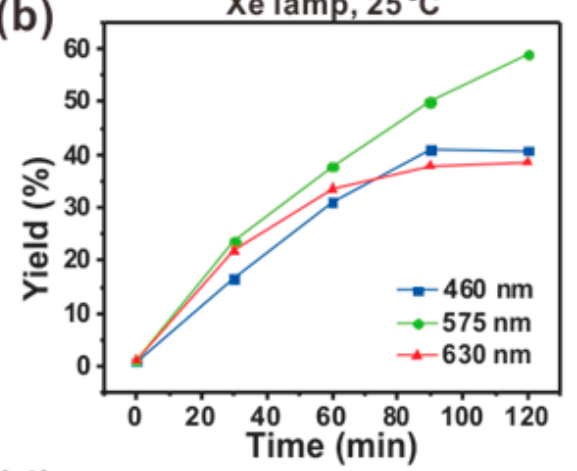

(d)

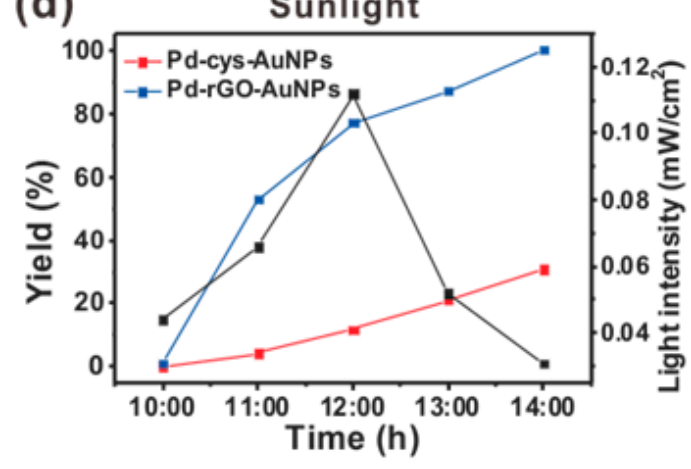

Figure 5. Catalytic performances of Pd-rGO-AuNPs for the Suzuki C-C coupling reaction at various conditions. (a) UV-Vis spectrum of Pd-rGO-AuNPs for excitation wavelength-dependent Suzuki-coupling reaction, (b) Wavelength-dependent Suzuki-coupling reaction (blue square, $460 \mathrm{~nm}$; green circle, $575 \mathrm{~nm}$; red triangle, $630 \mathrm{~nm}$ ), (c) Mechanism study in the presence of Pd-rGO-AuNPs with TEA, $\mathrm{KBrO}_{3}$ under Xe-lamp, $25^{\circ} \mathrm{C}$, (d) Suzuki-Miyaura cross-coupling reaction under sunlight illumination (black line: Changes of light intensity with time $\left(\mathrm{mW} / \mathrm{cm}^{2}\right)$ ).

\subsection{Suzuki-Miyaura Cross-Coupling Reaction under Sunlight}

The ultimate goal of using a visible-light induced photocatalyst is to employ sunlight as the energy source. The series of experiments performed indicated the interface-controlled $\mathrm{Pd}-\mathrm{Au}$ nanostructures (Pd-rGO-AuNPs) to be the most efficient photocatalyst. We investigated the capability of Pd-rGO-AuNPs for Suzuki-coupling reactions with sunlight. The Pd-rGO-AuNP solution (OD $0.4,5.0 \mathrm{~mL})$ mixed with phenylboronic acid $(97.6 \mathrm{mg}, 0.8 \mathrm{mmol})$, ethanol $(6.25 \mathrm{~mL})$, iodobenzene ( $44.5 \mu \mathrm{L}, 0.4 \mathrm{mmol})$, and $\mathrm{K}_{2} \mathrm{CO}_{3}(1.25 \mathrm{~mL}, 1 \mathrm{mmol})$ was exposed to sunlight at daytime (10:00-16:00, South Korea, Seoul) and stirred at ambient temperature by monitoring the reaction progress with high performance liquid chromatography (HPLC). In spite of the lower intensity of sunlight $\left(6.0 \mathrm{~mW} / \mathrm{cm}^{2}\right)$ as compared with that of the Xe lamp $\left(5.24 \mathrm{~W} / \mathrm{cm}^{2}\right)$, the coupling reaction was completed within $3 \mathrm{~h}$ 
of sunlight illumination. The final yield of the reaction was found to be $99 \%$ (analytical yield), and the solution temperature after $3 \mathrm{~h}$ was measured to be $37.1^{\circ} \mathrm{C}$. Exactly same reaction carried out with Pd-cys-AuNPs only resulted in the formation of the C-C coupling product with a low yield (31\%), which indicates the strong potential of interface engineering between the $\mathrm{Pd}-\mathrm{Au}$ nanostructure in obtaining improved photocatalytic activity.

\subsection{Photocatalytic Performance of Pd-rGO AuNPs for Various Aryl Halides and Substituted Phenyobronic Acid}

To investigate the photocatalytic performances of Pd-rGO-AuNPs to the different substrates for Suzuki-Miyaura C-C coupling reactions, a substituted phenylboronic acid and bromobenzene, chlorobenzene were reacted in the presence of Pd-rGO-AuNPs and Xe-lamp illuminations. The same reaction conditions performed in Section 2.6, but without controlling temperature, was used in this study and the results are summarized in Figure S12. The reaction between iodobenzene and phenylboronic acid containing an electron-donating group $\left(\mathrm{CH}_{3}\right)$ (p-tolylboronic acid) showed an excellent conversion yield $(99.2 \%)$ with a slightly decreased selectivity $(95.3 \%)$ for biphenyl product (Entry 2 in Figure S12). This is because of intrinsic self-coupling reaction of aryl boronic acid [36,37]. The self-coupling reaction in current conditions is more significant in the case of phenylbronic acid compared with that of iodobenzene (Figure S13). The cross-coupling reaction is not significant in the absence of Pd-rGO-AuNP catalyst (Figure S13). When a methyl group is present in the para-position of iodobenzene, there was also a slight decrease of conversion yield $(94.2 \%)$ and selectivity $(96.5 \%)$ (Entry 3 in Figure S12). Instead of iodobenzene, aryl halides such as bromobenzene and chlorobenzene were investigated for the coupling reactions. Similar to the reported low reactivity of bromo, chloro substituents, the photocatalytic reactions with Pd-rGO-AuNPs also showed decreased conversion yield $(75.5 \%, 31.1 \%$, respectively). These results are well matched with the recent results performed with heterogeneous visible-light induced photocatalysts.

\section{Materials and Methods}

\subsection{Materials}

Ethanol and acetonitrile were purchased from DAEJUNG (Siheung, South Korea). All other chemical reagents, $\mathrm{HAuCl}_{4}$, cysteamine, sodium borohyride $\left(\mathrm{NaBH}_{4}\right), \mathrm{K}_{2} \mathrm{PdCl}_{4}$, L-ascorbic acid, sodium hydroxide $(\mathrm{NaOH})$, polyvinylpyrrolidone (PVP), phenylboronic acid, iodobenzene, biphenyl, potassium carbonate $\left(\mathrm{K}_{2} \mathrm{CO}_{3}\right)$, triethanolamine (TEA), potassium bromate $\left(\mathrm{KBrO}_{3}\right)$, tetraethyl orthosilicate (TEOS), isopropyl alcohol, and sodium dodecylsulfate (SDS) were purchased from Sigma-Aldrich (St. Louis, MO, USA) and used as received without further purification.

\subsection{Synthesis of Cysteamine-Modified AuNPs (cys-AuNPs)}

To the aqueous solution of $\mathrm{HAuCl}_{4}(40 \mathrm{~mL}, 1.42 \mathrm{mM}), 400 \mu \mathrm{L}$ cysteamine $(213 \mu \mathrm{M})$ and $10 \mu \mathrm{L}$ cold $\left(\sim 0-4{ }^{\circ} \mathrm{C}\right)$ sodium borohyride $\left(\mathrm{NaBH}_{4}, 10 \mathrm{mM}\right)$ solutions were added with gentle stirring, and the mixture was then stirred for $12 \mathrm{~h}$ at room temperature [38]. The obtained purple solution was centrifuged at $2000 \mathrm{rcf}$ for $15 \mathrm{~min}$ to obtain the purified cys-AuNP solution $(30 \mathrm{~nm})$.

\subsection{Preparation of Nanosized Graphene Oxide (GO)}

GO was prepared using a modified Hummer's method [39]. A 9:1 mixture of concentrated $\mathrm{H}_{2} \mathrm{SO}_{4} / \mathrm{H}_{3} \mathrm{PO}_{4}(360: 40 \mathrm{~mL})$ was added to graphite flakes $(3.0 \mathrm{~g}) . \mathrm{KMnO}_{4}(18.0 \mathrm{~g})$ was added carefully while stirring, and the reaction mixture was then cooled in an ice bath at a temperature of $35-40{ }^{\circ} \mathrm{C}$. The reaction mixture was then heated to $50-55{ }^{\circ} \mathrm{C}$ and stirred for $12 \mathrm{~h}$. The reaction mixture was cooled to room temperature and poured into iced-water $(400 \mathrm{~mL})$ containing $30 \% \mathrm{H}_{2} \mathrm{O}_{2}(3.0 \mathrm{~mL})$. The solution color changed to yellow. The solution was then filtered through a metal US Standard testing sieve (W.S. Tyler, $300 \mu \mathrm{m}$ ). The filtrate was centrifuged (3000 rff for $30 \mathrm{~min}$ ) and the supernatant 
was decanted. The obtained gel-like product was then washed in succession with water $(200 \mathrm{~mL})$, $\mathrm{HCl}(30 \%, 200 \mathrm{~mL})$, ethanol $(200 \mathrm{~mL})$, and water until the $\mathrm{pH}$ of the solution was 5.0-6.0, and the solution was lyophilized for 3 days. GO powder $(5.0 \mathrm{mg})$ was dissolved in distilled water and exfoliated using probe sonication ( $35 \%$ amplitude, $500 \mathrm{~W}, 2.0 \mathrm{~h}$ ). The solution was centrifuged several times until no precipitate was observed $(15,000 \mathrm{rcf}, 20 \mathrm{~min})$. The size of the $\mathrm{GO}$ sheet was found to be less than $100 \mathrm{~nm}[38]$.

\subsection{Preparation of GO-Coated or rGO-Coated Gold Nanoparticles}

Firstly, $10 \mathrm{~mL}$ of the positively charged AuNPs (cys-AuNPs, particle size $30 \mathrm{~nm}$, optical density (OD) 1.0 at $530 \mathrm{~nm}$ ) were mixed with $10 \mathrm{~mL}$ of the negatively charged GO $(<100 \mathrm{~nm})$ solution $(0.25 \mathrm{mg} / \mathrm{mL})$, and the mixture was shaken for $12 \mathrm{~h}$ at room temperature. The mixture was centrifuged twice at 15,000 rcf for $15 \mathrm{~min}$ to remove excess GO from the precipitate (it should be noted that the nano-sized GO was not settled down at such conditions (15,000 rcf, $15 \mathrm{~min}$ ) because of its small size). The precipitated GO-coated AuNPs (GO-AuNPs) were then dispersed in distilled water (10 $\mathrm{mL})$ [38]. To prepare rGO-coated AuNPs (rGO-AuNPs), the GO-AuNP solution $(10 \mathrm{~mL})$ was reacted at $95-100{ }^{\circ} \mathrm{C}$ with ammonia solution $(250 \mu \mathrm{L}, 28 \%)$, hydrazine monohydrate $\left(50 \mu \mathrm{L}, \mathrm{NH}_{2} \mathrm{NH}_{2}-\mathrm{H}_{2} \mathrm{O}\right)$, and $1 \mu \mathrm{L}$ $1 \%$ SDS. The solution color changed from pale yellow brown to dark brown as the reaction progressed. To purify rGO-AuNPs from the reaction mixture, the solution was centrifuged twice at 15,000 rcf for $15 \mathrm{~min}$ and dispersed in distilled water [38].

3.5. Synthesis of Pd-Nanodot-Modified cys-AuNPs (Pd-cys-AuNPs), Pd-Nanodot-Modified GO-AuNPs (Pd-GO-AuNPs), and Pd-Nanodot-Modified rGO-AuNPs (Pd-rGO-AuNPs)

Pd precursor $\left(\mathrm{K}_{2} \mathrm{PdCl}_{4} \cdot \mathrm{H}_{2} \mathrm{O}\right)$ was reduced with ascorbic acid in the presence of cys-AuNPs, GO-AuNPs, or rGO-AuNPs. cys-AuNPs (10 mL, OD 1.0 at $530 \mathrm{~nm})$, GO-AuNPs (10 mL; OD 1.0 at $530 \mathrm{~nm})$, or rGO-AuNPs (10 mL; OD 1.2 at $530 \mathrm{~nm})$ were initially mixed with $5.0 \mathrm{~mL} \mathrm{1 \%} \mathrm{PVP}(5 \mathrm{~mL})$, ascorbic acid $(1.136 \mathrm{~mL}, 0.1 \mathrm{M})$, and $\mathrm{K}_{2} \mathrm{PdCl}_{4} \cdot \mathrm{H}_{2} \mathrm{O}(100 \mu \mathrm{L}, 0.01 \mathrm{M})$. The solutions were mixed by repeated gentle inversion for $10 \mathrm{~s}$ and left undisturbed at room temperature for $12 \mathrm{~h}$. The resulting solutions were centrifuged at 10,000 $\mathrm{rcf}$ for $15 \mathrm{~min}$ to purify the prepared nanoparticles, followed by re-dispersing the particles in distilled water $(10 \mathrm{~mL})$.

\subsection{Experimental Conditions for Kinetic Study}

The catalytic activity of the $\mathrm{Pd}-\mathrm{Au}$ catalyst with or without the graphene interface was quantitatively compared using a potassium hexacyanoferrate (III) $\left(\mathrm{Fe}^{3+}, 500 \mu \mathrm{M}\right)$ reduction model in the presence of Pd-cys-AuNPs $(10 \mathrm{~mL}, \mathrm{OD}$ at $530 \mathrm{~nm}=0.33$ or 0.40$)$ at room temperature [31]. The solution was purged with $\mathrm{N}_{2}$ and then irradiated with an Xe-lamp (power density: $5.24 \mathrm{~W} / \mathrm{cm}^{2}$ ) or an NIR laser $\left(808 \mathrm{~nm}, 11.4 \mathrm{~W} / \mathrm{cm}^{2}\right)$.

\subsection{Experimental Setup for Suzuki-Miyaura Cross-Coupling Reactions}

The Suzuki-Miyaura cross-coupling reaction between phenylboronic acid and iodobenzene was employed [40]. The nanoparticle solution $(10 \mathrm{~mL}, \mathrm{OD}$ at $540 \mathrm{~nm}=1.0)$ was placed in a reactor (Pyrex, window diameter $=11 \mathrm{~mm}$ ) equipped with a water circulation jacket (Figure S1). The solution was irradiated with an Xe lamp (power density: $5.24 \mathrm{~W} / \mathrm{cm}^{2}$ ) for $2 \mathrm{~h}$, and the amount of the product (biphenyl) obtained with Pd-rGO-AuNPs was monitored by employing HPLC analysis.

\subsection{Suzuki-Miyaura Cross-Coupling Reaction with Sunlight}

The Pd-rGO-AuNP solution (OD 0.4, $5.0 \mathrm{~mL}$ ) was mixed with phenylboronic acid $(97.6 \mathrm{mg}$, $0.8 \mathrm{mmol})$, ethanol $(6.25 \mathrm{~mL})$, iodobenzene $(44.5 \mu \mathrm{L}, 0.4 \mathrm{mmol})$, and $\mathrm{K}_{2} \mathrm{CO}_{3}(1.25 \mathrm{~mL}, 1 \mathrm{mmol})$ [40]. The mixture was exposed to sunlight on sunny days from 10:00 to 16:00 in February 2017 at ambient temperature. 


\subsection{Analytical Conditions to Monitor the Suzuki C-C Coupling Reaction}

An HPLC system equipped with a UV detector $(210 \mathrm{~nm})$ was used to monitor the reaction progress $(150 \mathrm{~mm} \times 3.0 \mathrm{~mm}, 3.5 \mu \mathrm{m}$ ZORBAX Eclipse AAA column, eleuent: 70\% acetonitrile aqueous solution, flow rate: $1.0 \mathrm{~mL} / \mathrm{min}$ ) [41]. The peak areas were calculated using a data analysis program (OpenLab ChemStation Edition for LC and LC/MS Systems, Agilent Technologies, Santa Clara, CA, USA).

\subsection{Characterization}

Transmission electron microscopy (TEM; H-7100, Hitachi, Tokyo, Japan) measurements were employed using the formvar/carbon coated copper grid (Ted Pella Inc., Redding, CA, USA). TEM (Titan, FEI Company, Eindhoven, The Netherlands) measurements using the holey copper grid (C-flat ${ }^{\circledR}$, Electron Microscopy Science, Hartfield, VA, USA) were employed for elemental mapping. Extinction spectra were obtained with a UV-visible spectrophotometer (Agilent, Santa Clara, CA, USA). Raman spectra analysis was carried out with a home-built Raman spectroscope equipped with a $532 \mathrm{~nm}$ laser system. Hydrodynamic size distribution was obtained with a particle size analyzer (ELSZ-1000, Otsuka Electronics, Osaka, Japan). An Xe lamp (Ceramaxs, Waltham, MA, USA) was used as the visible light (400-780 nm) source. In addition, a near-infrared (NIR) laser (Sanctity Laser SSL-808-6000-10TM-MF, Shanghai, China; $808 \mathrm{~nm}$ ) was used. The light intensity for all the light sources was measured using a laser power meter (THORLABS, Newton, NJ, USA). The amount of elements in the samples was measured with an induced coupled plasma (ICP)-mass instrument (Varian 820-MS, Victoria, Australia).

\section{Conclusions}

Interface-controlled Pd-nanodot-decorated AuNPs were easily synthesized using a wet-chemical reduction method by employing a Pd precursor in the presence of positively charged AuNPs or GO-coated AuNPs. We found that the presence of a graphene interface layer between the Pd-Au hetero-element is critical for obtaining the $\mathrm{C}-\mathrm{C}$ coupling product with a high yield with visible-light. The mechanistic study indicates that the electron-hole pair generated by the absorption of light energy is responsible for the enhanced photocatalytic activity. Although the photothermal effect could be a possible factor causing an increase in the yield of the $\mathrm{C}-\mathrm{C}$ coupling product, the experimental results show the efficient hot electron transfer from plasmonic nanoparticles to the active catalyst to be the key factor determining the plasmonic hybrid photocatalyst activity. The colloidal stability of the Pd-nanodot-modified AuNPs containing the graphene interface was excellent, and the photocatalytic activity of Pd-nanodot-modified AuNPs with graphene interface was maintained after performing the C-C coupling reactions (Figure S14). This new finding highlights the potential of interface engineering in the design of plasmonic nanoparticle-based visible-light-induced photocatalysts.

Supplementary Materials: The following are available online at http:/ /www.mdpi.com/2073-4344/8/10/463/s1, Figure S1. Instrumental setup for the Suzuki-Miyaura coupling reaction. (a) Light from the Xe lamp (400-780 nm) illuminates the reactor (Pyrex, $15 \mathrm{~mL}$ ) equipped with a water circulation jacket. (b) The emission profiles of Xe-lamp used in this experiment; Figure S2. (a) Preparation of Pd-nanodot-decorated AuNPs using cysteamine-modified AuNPs with or without $1 \%$ PVP, (b) UV-Vis spectra of Pd-cys-AuNPs prepared with (blue-line) or without PVP (red-line), (c) TEM images of the nanoparticles prepared without 1\% PVP, (d) TEM images of the nanoparticles prepared with 1\% PVP; Figure S3. (a) Preparation of Pd-nanodot-decorated AuNPs using citrate-modified AuNPs with or without 1\% PVP, (b) UV-Vis spectra of Pd-citrate-AuNPs prepared with (blue-line) or without PVP (red-line), (c) TEM images of the nanoparticles prepared without 1\% PVP, (d) TEM images of the nanoparticles prepared with 1\% PVP; Figure S4. TEM images of (a) Pd-cys-AuNPs, (b) Pd-GO-AuNPs, and (c) Pd-rGO-AuNPs (Scale bar in the inset is $10 \mathrm{~nm}$ ); Figure S5. (a) XRD spectra and size distribution of (b) Pd-cys-AuNPs, (c) Pd-GO-AuNPs, and (d) Pd-rGO-AuNPs; Figure S6. Time dependent reduction of $\mathrm{Fe}^{3+}$ to $\mathrm{Fe}^{2+}$ in the presence of (a) Pd-cys-AuNPs, (b) Pd-GO-AuNPs, and (c) Pd-rGO-AuNPs with Xe lamp illumination; Figure S7. Time dependent reduction of $\mathrm{Fe}^{3+}$ to $\mathrm{Fe}^{2+}$ in the presence of (a) Pd-cys-AuNPs, (b) Pd-GO-AuNPs, and (c) Pd-rGO-AuNPs with NIR laser; Figure S8. Wavelength dependent reduction of $\mathrm{Fe}^{3+}$ to $\mathrm{Fe}^{2+}$ in the presence of Pd-rGO-AuNPs with Xe lamp illumination and band-pass filter (450, 500, and $650 \mathrm{~nm}$ ); Figure S9. Results of photocatalytic reactions performed in the presence of (a) Pd-cys AuNPs, (b) Pd-GO-AuNPs, or (c) Pd-rGO-AuNPs at uncontrolled temperature (Entry $1,2,3)$ or at controlled temperature $\left(25^{\circ} \mathrm{C}\right)($ Entry $4,5,6)$ and calibration curve for biphenyl product; Figure S10. The representative chromatogram of the Suzuki-coupling reaction (Retention 
time: by product $-0.5 \mathrm{~min}$, phenyl boronic acid $-0.7 \mathrm{~min}$, iodobenzene $-1.9 \mathrm{~min}$, biphenyl (product)—2.3 min); Figure S11. Temperature changes of reaction mixtures during the coupling reactions with light illumination in the presence of Pd-cys-AuNPs, Pd-GO-AuNPs, and Pd-rGO-AuNPs; Figure S12. The summary of coupling reaction with various haloarens and substituted phenylbronic acids in the presence of Pd-rGO-AuNPs with light illumination; Figure S13. The results of self-coupling reaction with phenylboronic acid (a) or iodobenzene (b) in the presence of Pd-rGO-AuNPs with light illumination. The visible light induced reaction without Pd-rGO-AuNPs catalyst (c); Figure S14. Comparison of Pd-rGO-AuNPs before and after the Suzuki-Miyaura coupling reaction. (a) UV-Vis spectra of Pd-rGO-AuNPs before (red line) and after reaction (green line), (b) TEM image of Pd-rGO-AuNPs after reaction (Scale bar in the inset is $20 \mathrm{~nm}$ ). (c) The chromatogram of reaction mixtures after completion of reaction using fresh Pd-rGO-AuNP catalyst. (d) The chromatogram of reaction mixtures after completion of reaction using recycled Pd-rGO-AuNP catalyst.

Author Contributions: Conceptualization, D.-K.L.; methodology, E.K.; validation, E.K. and H.H.S.; formal analysis, E.K. and H.H.S.; investigation, E.K. and H.H.S.; writing-original draft preparation, D.-K.L. and E.K.; writing-review and editing, D.-K.L.; supervision, D.-K.L.; project administration, D.-K.L.; funding acquisition, D.-K.L.

Funding: This work was also supported by national research fund (2018R1A2A3075499) and KU-KIST research fund.

Conflicts of Interest: The authors declare no conflict of interest.

\section{References}

1. Miyaura, N.; Suzuki, A. Palladium-catalyzed cross-coupling reactions of organoboron compounds. Chem. Rev. 1995, 95, 2457-2483. [CrossRef]

2. Molnár, A.R.D. Efficient, selective, and recyclable palladium catalysts in carbon-carbon coupling reactions. Chem. Rev. 2011, 111, 2251-2320. [CrossRef] [PubMed]

3. Glasspoole, B.W.; Crudden, C.M. Cross-coupling: The final frontier. Nat. Chem. 2011, 3, 912-913. [CrossRef] [PubMed]

4. Fihri, A.; Bouhrara, M.; Nekoueishahraki, B.; Basset, J.-M.; Polshettiwar, V. Nanocatalysts for Suzuki cross-coupling reactions. Chem. Soc. Rev. 2011, 40, 5181-5203. [CrossRef] [PubMed]

5. Miyaura, N.; Yamada, K.; Suzuki, A. A new stereospecific cross-coupling by the palladium-catalyzed reaction of 1-alkenylboranes with 1-alkenyl or 1-alkynyl halides. Tetrahedron Lett. 1979, 20, 3437-3440. [CrossRef]

6. Ngnie, G.; Dedzo, G.K.; Detellier, C. Synthesis and catalytic application of palladium nanoparticles supported on kaolinite-based nanohybrid materials. Dalton Trans. 2016, 45, 9065-9072. [CrossRef] [PubMed]

7. Choi, H.R.; Woo, H.; Jang, S.; Cheon, J.Y.; Kim, C.; Park, J.; Park, K.H.; Joo, S.H. Ordered mesoporous carbon supported colloidal Pd nanoparticle based model catalysts for Suzuki coupling reactions: Impact of organic capping agents. ChemCatChem 2012, 4, 1587-1594. [CrossRef]

8. Magne, O.S. Use of nanoparticles as catalysts in organic synthesis-cross-coupling reactions. Curr. Org. Chem. 2014, 18, 312-326.

9. Mora, M.; Jimenez-Sanchidrian, C.; Rafael Ruiz, J. Recent advances in the heterogeneous palladium-catalysed Suzuki cross-coupling reaction. Curr. Org. Chem. 2012, 16, 1128-1150. [CrossRef]

10. Clavero, C. Plasmon-induced hot-electron generation at nanoparticle/metal-oxide interfaces for photovoltaic and photocatalytic devices. Nat. Photonics 2014, 8, 95-103. [CrossRef]

11. Shaw, M.H.; Twilton, J.; MacMillan, D.W.C. Photoredox catalysis in organic chemistry. J. Org. Chem. 2016, 81, 6898-6926. [CrossRef] [PubMed]

12. Nicewicz, D.A.; MacMillan, D.W. Merging photoredox catalysis with organocatalysis: The direct asymmetric alkylation of aldehydes. Science 2008, 322, 77-80. [CrossRef] [PubMed]

13. Tucker, J.W.; Stephenson, C.R.J. Shining light on photoredox catalysis: Theory and synthetic applications. J. Org. Chem. 2012, 77, 1617-1622. [CrossRef] [PubMed]

14. Martina, K.; Manzoli, M.; Gaudino, E.C.; Cravotto, G. Eco-friendly physical activation methods for Suzuki-Miyaura reactions. Catalysts 2017, 7, 98. [CrossRef]

15. Knight, M.W.; Wang, Y.; Urban, A.S.; Sobhani, A.; Zheng, B.Y.; Nordlander, P.; Halas, N.J. Embedding plasmonic nanostructure diodes enhances hot electron emission. Nano Lett. 2013, 13, 1687-1692. [CrossRef] [PubMed]

16. Atwater, H.A.; Polman, A. Plasmonics for improved photovoltaic devices. Nat. Mater. 2010, 9, $205-213$. [CrossRef] [PubMed] 
17. Linic, S.; Christopher, P.; Ingram, D.B. Plasmonic-metal nanostructures for efficient conversion of solar to chemical energy. Nat. Mater. 2011, 10,911-921. [CrossRef] [PubMed]

18. Zheng, Z.; Tachikawa, T.; Majima, T. Single-particle study of Pt-modified Au nanorods for plasmon-enhanced hydrogen generation in visible to near-infrared region. J. Am. Chem. Soc. 2014, 136, 6870-6873. [CrossRef] [PubMed]

19. Brongersma, M.L.; Halas, N.J.; Nordlander, P. Plasmon-induced hot carrier science and technology. Nat. Nanotechnol. 2015, 10, 25-34. [CrossRef] [PubMed]

20. Mori, K.; Kawashima, M.; Yamashita, H. Visible-light-enhanced Suzuki-Miyaura coupling reaction by cooperative photocatalysis with an Ru-Pd bimetallic complex. Chem. Commun. 2014, 50, 14501-14503. [CrossRef] [PubMed]

21. Zhang, S.; Chang, C.; Huang, Z.; Ma, Y.; Gao, W.; Li, J.; Qu, Y. Visible-Light-Activated Suzuki-Miyaura Coupling Reactions of Aryl Chlorides over the Multifunctional Pd/Au/Porous Nanorods of $\mathrm{CeO}_{2}$ Catalysts. ACS Catal. 2015, 5, 6481-6488. [CrossRef]

22. Zheng, B.Y.; Zhao, H.; Manjavacas, A.; McClain, M.; Nordlander, P.; Halas, N.J. Distinguishing between plasmon-induced and photoexcited carriers in a device geometry. Nat. Commun. 2015, 6, 7797. [CrossRef] [PubMed]

23. Hoggard, A.; Wang, L.-Y.; Ma, L.; Fang, Y.; You, G.; Olson, J.; Liu, Z.; Chang, W.-S.; Ajayan, P.M.; Link, S. Using the plasmon linewidth to calculate the time and efficiency of electron transfer between gold nanorods and graphene. ACS Nano 2013, 7, 11209-11217. [CrossRef] [PubMed]

24. Knight, M.W.; Sobhani, H.; Nordlander, P.; Halas, N.J. Photodetection with active optical antennas. Science 2011, 332, 702-704. [CrossRef] [PubMed]

25. DuChene, J.S.; Sweeny, B.C.; Johnston-Peck, A.C.; Su, D.; Stach, E.A.; Wei, W.D. Prolonged hot electron dynamics in plasmonic-metal/semiconductor heterostructures with implications for solar photocatalysis. Angew. Chem. Int. Ed. 2014, 53, 7887-7891. [CrossRef] [PubMed]

26. Mubeen, S.; Lee, J.; Singh, N.; Kramer, S.; Stucky, G.D.; Moskovits, M. An autonomous photosynthetic device in which all charge carriers derive from surface plasmons. Nat. Nanotechnol. 2013, 8, 247-251. [CrossRef] [PubMed]

27. Kumar, D.; Lee, A.; Lee, T.; Lim, M.; Lim, D.-K. Ultrafast and efficient transport of hot plasmonic electrons by graphene for Pt free, highly efficient visible-light responsive photocatalyst. Nano Lett. 2016, 16, 1760-1767. [CrossRef] [PubMed]

28. Chen, J.-H.; Jang, C.; Xiao, S.; Ishigami, M.; Fuhrer, M.S. Intrinsic and extrinsic performance limits of graphene devices on $\mathrm{SiO}_{2}$. Nat. Nanotechnol. 2008, 3, 206-209. [CrossRef] [PubMed]

29. Nieboer, E.; Mcbryde, W.A.E. Free-energy relationships in coordination chemistry. III. A Comprehensive. Can. J. Chem. 1972, 51, 2512-2524. [CrossRef]

30. Kumar, D.; Kaur, S.; Lim, D.-K. Plasmon-assisted and visible-light induced graphene oxide reduction and efficient fluorescence quenching. Chem. Commun. 2014, 50, 13481-13484. [CrossRef] [PubMed]

31. Kim, Y.; Dumett Torres, D.; Jain, P.K. Activation Energies of Plasmonic Catalysts. Nano Lett. 2016, 16, 3399-3407. [CrossRef] [PubMed]

32. Yen, C.-W.; El-Sayed, M.A. Plasmonic field effect on the hexacyanoferrate (III)-thiosulfate electron transfer catalytic reaction on gold nanoparticles: Electromagnetic or thermal? J. Phys. Chem. C 2009, 113, 19585-19590. [CrossRef]

33. Adleman, J.R.; Boyd, D.A.; Goodwin, D.G.; Psaltis, D. Heterogenous catalysis mediated by plasmon heating. Nano Lett. 2009, 9, 4417-4423. [CrossRef] [PubMed]

34. Gomes Silva, C.U.; Juárez, R.; Marino, T.; Molinari, R.; García, H. Influence of excitation wavelength (UV or visible light) on the photocatalytic activity of titania containing gold nanoparticles for the generation of hydrogen or oxygen from water. J. Am. Chem. Soc. 2010, 133, 595-602. [CrossRef] [PubMed]

35. Naya, S.-I.; Inoue, A.; Tada, H. Self-assembled heterosupramolecular visible light photocatalyst consisting of gold nanoparticle-loaded titanium (IV) dioxide and surfactant. J. Am. Chem. Soc. 2010, 132, 6292-6293. [CrossRef] [PubMed]

36. Shin, H.H.; Kang, E.; Park, H.; Han, T.; Lee, C.-H.; Lim, D.-K. Pd-nanodot decorated MoS2 nanosheets as a highly efficient photocatalyst for the visible-light-induced Suzuki-Miyaura coupling reaction. J. Mater. Chem. A 2017, 5, 24965-24971. [CrossRef] 
37. Moreno-Mañas, M.; Pérez, M.; Pleixats, R. Palladium-catalyzed Suzuki-type self-coupling of arylboronic acids. A mechanistic study. J. Org. Chem. 1996, 61, 2346-2351. [CrossRef]

38. Lim, D.-K.; Barhoumi, A.; Wylie, R.G.; Reznor, G.; Langer, R.S.; Kohane, D.S. Enhanced photothermal effect of plasmonic nanoparticles coated with reduced graphene oxide. Nano Lett. 2013, 13, 4075-4079. [CrossRef] [PubMed]

39. Marcano, D.C.; Kosynkin, D.V.; Berlin, J.M.; Sinitskii, A.; Sun, Z.; Slesarev, A.; Alemany, L.B.; Lu, W.; Tour, J.M. Improved synthesis of graphene oxide. ACS Nano 2010, 4, 4806-4814. [CrossRef] [PubMed]

40. Li, X.-H.; Baar, M.; Blechert, S.; Antonietti, M. Facilitating room-temperature Suzuki coupling reaction with light: Mott-Schottky photocatalyst for CC-coupling. Sci. Rep. 2013, 3, 1743. [CrossRef]

41. Scheuermann, G.M.; Rumi, L.; Steurer, P.; Bannwarth, W.; Mülhaupt, R. Palladium nanoparticles on graphite oxide and its functionalized graphene derivatives as highly active catalysts for the Suzuki-Miyaura coupling reaction. J. Am. Chem. Soc. 2009, 131, 8262-8270. [CrossRef] [PubMed]

(C) 2018 by the authors. Licensee MDPI, Basel, Switzerland. This article is an open access article distributed under the terms and conditions of the Creative Commons Attribution (CC BY) license (http://creativecommons.org/licenses/by/4.0/). 